\title{
Entropy and Channel Capacity under Optimum Power and Rate Adaptation over Generalized Fading Conditions
}

\author{
Paschalis C. Sofotasios, Member, IEEE, Sami Muhaidat, Senior Member, IEEE, Mikko Valkama, Member, IEEE, \\ Mounir Ghogho, Senior Member, IEEE, and George K. Karagiannidis, Fellow, IEEE
}

\begin{abstract}
Accurate fading characterization and channel capacity determination are of paramount importance in both conventional and emerging communication systems. The present work addresses the non-linearity of the propagation medium and its effects on the channel capacity. Such fading conditions are first characterized using information theoretic measures, namely, Shannon entropy, cross entropy and relative entropy. The corresponding effects on the channel capacity with and without power adaptation are then analyzed. Closed-form expressions are derived and validated through computer simulations. It is shown that the effects of nonlinearities are significantly larger than those of fading parameters such as the scattered-wave power ratio, and the correlation coefficient between the in-phase and quadrature components in each cluster of multipath components.
\end{abstract}

Index Terms-Adaptation policies, channel capacity, entropy.

\section{INTRODUCTION}

$\mathbf{M}$ ULTIPATH fading characterization is considered a critical task in the effective analysis of the performance of wireless communication systems. To this end, the $\alpha-\mu, \eta-\mu$,

Manuscript received April 09, 2015; revised June 16, 2015; accepted June 26, 2015. Date of publication August 04, 2015; date of current version August 13, 2015. This work was supported by the Finnish Funding Agency for Technology and Innovation (Tekes) under the project entitled "Energy-Efficient Wireless Networks and Connectivity of Devices-Systems (EWINE-S)" and by the Academy of Finland under the project No. 284694 "Fundamentals of Ultra Dense 5G Networks with Application to Machine Type Communication." The associate editor coordinating the review of this manuscript and approving it for publication was Prof. Qian He.

P. C. Sofotasios is with the Department of Electronics and Communications Engineering, Tampere University of Technology, 33101 Tampere, Finland, and also with the Department of Electrical and Computer Engineering, Aristotle University of Thessaloniki, 54124 Thessaloniki, Greece (e-mail p.sofotasios@ieee.org).

S. Muhaidat is with the Department of Electrical and Computer Engineering, Khalifa University, Abu Dhabi, UAE, and also with the Centre for Communication Systems Research, Department of Electronic Engineering, University of Surrey, GU2 7XH Guildford, U.K. (e-mail: muhaidat@ieee.org).

M. Valkama is with the Department of Electronics and Communications Engineering, Tampere University of Technology, 33101 Tampere, Finland (e-mail: mikko.e.valkama@tut.fi).

M. Ghogho is with the School of Electronic and Electrical Engineering, University of Leeds, LS2 9JT Leeds, U.K., and also with the International University of Rabat, 10100 Rabat, Morocco (e-mail: m.ghogho@ieee.org).

G. K. Karagiannidis is with the Department of Electrical and Computer Engineering, Aristotle University of Thessaloniki, 54124 Thessaloniki, Greece, and also with the Department of Electrical and Computer Engineering, Khalifa University, 127788 Abu Dhabi, UAE (e-mail: geokarag@ieee.org).

Color versions of one or more of the figures in this paper are available online at http://ieeexplore.ieee.org. $\lambda-\mu$ and $\kappa-\mu$ distributions have been considered relatively suitable models as they also include as special cases the widely known Rayleigh, Nakagami $-m$, Hoyt, Weibull and Rice distributions, see [1]-[21] and the references therein.

It is also known that accurate determination of the channel capacity for different communication scenarios has been of core importance in wireless communications, including requirements on optimum rate and transmit power constraints. To this end, the average channel capacity over generalized fading channels was thoroughly analyzed in [4], whereas the channel capacity under different adaptation policies was thoroughly investigated in [22]-[24].

Nevertheless, in spite of the usefulness of the aforementioned distributions, their accuracy and generality are limited, since the non-linearity parameter $\alpha$ in these models is not related to the fading parameters $\eta, \lambda$ and $\kappa$ [25]. As a consequence, the detrimental effects of non-linear propagation medium are not considered in the majority of investigations. Motivated by this, the authors in [25] proposed the $\alpha-\eta-\mu$ and $\alpha-\kappa-\mu$ distributions which constitute a generalization of the $\alpha-\mu, \eta-\mu$ and $\kappa-\mu$ models and were shown to provide remarkably accurate fitting to measurement results in various communication scenarios. In this context, useful statistical results and properties for the $\alpha-\lambda-\mu$ and $\alpha-\eta-\mu$ models were reported in [26]-[28] whereas the corresponding outage probability was analyzed in [29]. A simple and remarkably accurate random sequence generator for $\alpha-\kappa-\mu$ and $\alpha-\eta-\mu$ variates was proposed in [30] while the corresponding symbol error rate and channel capacity was addressed in [31], [32].

However, none of the reported analyses address the corresponding channel capacity with optimum power and rate adaptation. Motivated by this, the aim of the present work is twofold: we firstly derive novel analytic expressions for the Shannon entropy of $\alpha-\eta-\mu$ and $\alpha-\lambda-\mu$ distributions. Capitalizing on this, we derive analytic expressions for the cross-entropy and relative entropy for these distributions with respect to the $\eta-\mu$ and $\lambda-\mu$ distributions, respectively. Secondly, we derive novel closed-form expressions for the corresponding channel capacity under optimum rate adaptation as well as optimum power and rate adaptation. The derived expressions are subsequently used in evaluating the corresponding performance, which indicates that the effects of fading non-linearities are larger than those of popular and widely considered fading parameters. 


\section{NON-LINEAR FADING CHANNELS AND ENTROPIES}

A. The $\alpha-\eta-\mu$ and $\alpha-\lambda-\mu$ Models

The SNR PDF of the $\alpha-\eta-\mu$ distribution is given by [25]

$$
\begin{aligned}
& p_{\gamma}(\gamma) \\
& =\frac{\alpha \sqrt{\pi} \mu^{\mu+\frac{1}{2}}(\eta+1)^{\mu+\frac{1}{2}} \gamma^{\frac{\alpha \mu}{2}+\frac{\alpha}{4}-1} I_{\mu-\frac{1}{2}}\left(\frac{\left(\eta^{2}-1\right) \mu \gamma^{\frac{\alpha}{2}}}{2 \eta \bar{\gamma}^{\frac{\alpha}{2}}}\right)}{2 \sqrt{\eta} \Gamma(\mu)(\eta-1)^{\mu-\frac{1}{2}} \bar{\gamma}^{\frac{\alpha \mu}{2}+\frac{\alpha}{4}} \exp \left(\frac{(1+\eta)^{2} \mu \gamma^{\frac{\alpha}{2}}}{2 \eta \bar{\gamma}^{\frac{\alpha}{2}}}\right)}
\end{aligned}
$$

where $\alpha$ denotes the non-linearity of the propagation medium, $\eta$ is the scattered wave power ratio between the in-phase and quadrature components and $\mu$ is related to the number of multipath clusters. Likewise, the SNR PDF of the $\alpha-\lambda-\mu$ fading model is expressed as follows:

$$
p_{\gamma}(\gamma)=\frac{(-1)^{\mu-\frac{1}{2}} \alpha \sqrt{\pi} \mu^{\mu+\frac{1}{2}}}{\Gamma(\mu) \lambda^{\mu-\frac{1}{2}} \sqrt{1-\lambda^{2}}} \frac{\gamma^{\frac{\alpha \mu}{2}+\frac{\alpha}{4}-1}}{\gamma^{\frac{\alpha \mu}{2}+\frac{\alpha}{4}}} \frac{I_{\mu-\frac{1}{2}}\left(\frac{2 \lambda \mu \gamma^{\frac{\alpha}{2}}}{\left(\lambda^{2}-1\right) \bar{\gamma}^{\frac{\alpha}{2}}}\right)}{\exp \left(\frac{2 \mu \gamma^{\frac{\alpha}{2}}}{\left(1-\lambda^{2}\right) \bar{\gamma}^{\frac{\alpha}{2}}}\right)}
$$

where $\lambda$ denotes the correlation coefficient between the in-phase and quadrature components of the fading signal [26]. It is recalled that the $\alpha-\eta-\mu$ and $\alpha-\lambda-\mu$ distributions include as special cases the $\alpha-\mu$, the $\eta-\mu$ and the $\lambda-\mu$ fading models for $\alpha=2, \eta=2$ and $\lambda=0$, respectively [25].

\section{B. Shannon Entropy}

The Shannon entropy is a fundamental metric which denotes the amount of information contained in a signal and indicates the number of bits required for encoding this signal.

Lemma 1: For $\{\alpha, \eta, \gamma, \bar{\gamma}\} \in \mathbb{R}^{+}$and $\mu=1$, the Shannon entropy of the $\alpha-\eta-\mu$ fading distribution is expressed as

$$
\begin{array}{r}
H(p)=\frac{\mathcal{B}_{1}}{\alpha \ln (2)}\left\{\frac{\gamma^{\prime}+\ln \left(\mathcal{C}_{1}+\mathcal{D}_{1}\right)}{\sqrt{2 \pi \mathcal{D}_{1}}\left(\mathcal{C}_{1}+\mathcal{D}_{1}\right)}-\frac{\gamma^{\prime}+\ln \left(\mathcal{C}_{1}-\mathcal{D}_{1}\right)}{\sqrt{2 \pi \mathcal{D}_{1}}\left(\mathcal{C}_{1}-\mathcal{D}_{1}\right)}\right\} \\
+\frac{\mathcal{B}_{1}(3 \alpha-4)}{\ln (2) \alpha^{2} \sqrt{\pi}}\left\{\frac{\gamma^{\prime}+\ln \left(\mathcal{C}_{1}-\mathcal{D}_{1}\right)}{\sqrt{2 \mathcal{D}_{1}}\left(\mathcal{C}_{1}-\mathcal{D}_{1}\right)}-\frac{\gamma^{\prime}+\ln \left(\mathcal{C}_{1}+\mathcal{D}_{1}\right)}{\sqrt{2 \mathcal{D}_{1}}\left(\mathcal{C}_{1}+\mathcal{D}_{1}\right)}\right\} \\
+\frac{\mathcal{B}_{1} \sqrt{2}}{\ln (2) \alpha}\left\{\frac{\ln \left(\mathcal{B}_{1}\right)-\ln \left(\sqrt{2 \pi \mathcal{D}_{1}}\right)}{\sqrt{\pi} \mathcal{D}_{1}\left(\mathcal{C}_{1}+\mathcal{D}_{1}\right)}+\frac{\mathcal{D}_{1}-\mathcal{C}_{1} \mathcal{D}_{1}^{-1}}{\sqrt{\pi}\left(\mathcal{C}_{1}+\mathcal{D}_{1}\right)^{2}}\right. \\
\left.-\frac{\ln \left(\mathcal{B}_{1}\right)-\ln \left(\sqrt{2 \pi \mathcal{D}_{1}}\right)}{\sqrt{\pi} \mathcal{D}_{1}\left(\mathcal{C}_{1}-\mathcal{D}_{1}\right)}-\frac{\mathcal{D}_{1}-\mathcal{C}_{1} \mathcal{D}_{1}^{-1}}{\sqrt{\pi}\left(\mathcal{C}_{1}-\mathcal{D}_{1}\right)^{2}}\right\}
\end{array}
$$

in bits/message where

$$
\mathcal{B}_{1}=\frac{\alpha \sqrt{\pi}(\eta+1)^{\frac{3}{2}}}{2 \sqrt{\eta} \sqrt{(\eta-1)} \bar{\gamma}^{\frac{3 \alpha}{4}}}
$$

$\mathcal{C}_{1}=(1+\eta)^{2} /\left(2 \eta \bar{\gamma}^{\frac{a}{2}}\right)$ and $\mathcal{D}_{1}=\left(\eta^{2}-1\right) /\left(2 \eta \bar{\gamma}^{\frac{\alpha}{2}}\right)$ with $\gamma^{\prime}=$ 0.5772 denoting the Euler-Mascheroni constant.

Proof: The Shannon entropy for continuous random variables with PDF $p(x)$ is given by $H(p) \triangleq-\int_{0}^{\infty} p(x) \log _{2}(p(x)) \mathrm{d} x$. Thus, for the case of $\alpha-\eta-\mu$ fading in (1) and with the aid of standard logarithmic identities it follows that

$$
\begin{aligned}
H(p)= & \frac{\mathcal{B}_{1}}{\ln (2)} \int_{0}^{\infty}\left\{\frac{\mathcal{C}_{1}}{\gamma^{1-\frac{5 \alpha}{4}}}-\frac{\ln \left(\mathcal{B}_{1}\right)}{\gamma^{1-\frac{3 \alpha}{4}}}\right\} \frac{I_{\frac{1}{2}}\left(\mathcal{D}_{1} \gamma^{\frac{\alpha}{2}}\right)}{e^{\mathcal{C}_{1} \gamma^{\frac{\alpha}{2}}}} \mathrm{~d} \gamma \\
& -\frac{\mathcal{B}_{1}(3 \alpha-4)}{4 \ln (2)} \int_{0}^{\infty} \frac{\gamma^{\frac{3 \alpha}{4}-1} \ln (\gamma) I_{\frac{1}{2}}\left(\mathcal{D}_{1} \gamma^{\frac{\alpha}{2}}\right)}{e^{\mathcal{C}_{1} \gamma^{\frac{\alpha}{2}}}} \mathrm{~d} \gamma \\
& -\frac{\mathcal{B}_{1}}{\ln (2)} \int_{0}^{\infty} \frac{I_{\frac{1}{2}}\left(\mathcal{D}_{1} \gamma^{\frac{\alpha}{2}}\right) \ln \left[I_{\frac{1}{2}}\left(\mathcal{D}_{1} \gamma^{\frac{\alpha}{2}}\right)\right]}{\gamma^{1-\frac{3 \alpha}{4}} e^{\mathcal{C}_{1} \gamma^{\frac{\alpha}{2}}}} \mathrm{~d} \gamma
\end{aligned}
$$

By recalling that $I_{0.5}(x)=\left(e^{x}-e^{-x}\right) / \sqrt{2 \pi x}$, one obtains

$$
\begin{aligned}
H(p)= & \mathcal{B}_{1}\left[\ln \left(\mathcal{C}_{1}\right)-\mathcal{C}_{1}\right] \int_{0}^{\infty} \frac{e^{-\frac{\gamma^{a}}{2}\left(\mathcal{C}_{1}+\mathcal{D}_{1}\right)}-e^{-\frac{\gamma^{a}}{2}\left(\mathcal{C}_{1}-\mathcal{D}_{1}\right)}}{\ln (2) \sqrt{2 \pi \mathcal{D}_{1}} \gamma^{1-\frac{\alpha}{2}}} \mathrm{~d} \gamma \\
& +\frac{\mathcal{B}_{1}(3 \alpha-4)}{4 \ln (2) \sqrt{2 \pi \mathcal{D}_{1}}} \int_{0}^{\infty} \frac{e^{-\gamma^{\frac{\alpha}{2}}\left(\mathcal{C}_{1}+\mathcal{D}_{1}\right)}-e^{-\gamma^{\frac{\alpha}{2}}\left(\mathcal{C}_{1}-\mathcal{D}_{1}\right)}}{\gamma^{1-\frac{\alpha}{2}}[\ln (\gamma)]^{-1}} \mathrm{~d} \gamma \\
& +\mathcal{B}_{1} \int_{0}^{\infty} \frac{\gamma^{\frac{\alpha}{2}-1}\left\{e^{-\gamma^{\frac{\alpha}{2}}\left(\mathcal{C}_{1}+\mathcal{D}_{1}\right)}-e^{-\gamma^{\frac{\alpha}{2}}\left(\mathcal{C}_{1}-\mathcal{D}_{1}\right)}\right\}}{\ln \left(\frac{e^{\mathcal{D}_{1} \gamma^{\frac{\alpha}{2}}}-e^{-\mathcal{D}_{1} \gamma^{\frac{\alpha}{2}}}}{\sqrt{2 \pi \gamma^{\frac{\alpha}{2}}}}\right)^{-1} \ln (2) \sqrt{2 \pi \mathcal{D}_{1}}} \mathrm{~d} \gamma
\end{aligned}
$$

The first two integrals in (6) can be expressed in closed-form with the aid of [33, eq. (4.352.1)] and [33, eq. (8.310.1)] and involve the digamma function, $\psi(1)$. By also noticing that $\exp (-x)$ in the logarithm of (6) becomes practically negligible as $x \gg 0$, recalling that $\psi(1)=-\gamma^{\prime}$ and after some algebraic manipulations, one obtains (3), which completes the proof.

Importantly, the Shannon entropy can quantify the detrimental effects of fading non-linearity by determining the variation of required bits per message for each value of $\alpha$. This demonstrates the difference between the $\alpha-\eta-\mu$ and $\alpha-\lambda-\mu$ distributions with the popular $\eta-\mu$ and $\lambda-\mu$ distributions, respectively, which is also reflected by the considered channel capacity measures. This is subsequently analyzed along with the derivation of useful analytic expressions for the corresponding cross entropy and relative entropy metrics.

\section{Cross Entropy}

The cross entropy measures the average number of bits required to encode a message when a distribution $p(x)$ is replaced by a distribution $q(x)$. In the present analysis this can exhibit the number of bits required to encode $\alpha-\eta-\mu$ and $\alpha-\lambda-\mu$ when they are replaced by $\eta-\mu$ and $\lambda-\mu$ distributions, respectively, which corresponds to the case where the non-linearity parameter is not taken into account.

Lemma 2: For $\left\{\alpha, \eta, \eta \prime, \gamma, \bar{\gamma}, \bar{\gamma}^{\prime}\right\} \in \mathbb{R}^{+}$and $\mu=1$, the cross entropy between $\alpha-\eta-\mu$ and $\eta-\mu$ distributions is given by

$$
\begin{aligned}
& H(p, q) \\
& =\frac{\ln \left(\mathcal{B}_{2}\right)-\ln \left(\sqrt{2 \pi \mathcal{D}_{2}}\right)}{\mathcal{B}_{1}^{-1} 2^{-\frac{1}{2}} \alpha \ln (2) \sqrt{\pi \mathcal{D}_{1}}}\left\{\frac{1}{\mathcal{C}_{1}+\mathcal{D}_{1}}-\frac{1}{\mathcal{C}_{1}-\mathcal{D}_{1}}\right\} \\
& \quad+\frac{\sqrt{2}\left(\mathcal{C}_{2}-\mathcal{D}_{2}\right)}{\alpha \ln (2) \sqrt{\pi \mathcal{D}_{1}}}\left\{\frac{\mathcal{B}_{1} \Gamma\left(\frac{2}{\alpha}-1\right)}{\left(\mathcal{C}_{1}-\mathcal{D}_{1}\right)^{1+\frac{2}{a}}}-\frac{\mathcal{B}_{1} \Gamma\left(\frac{2}{\alpha}-1\right)}{\left(\mathcal{C}_{1}+\mathcal{D}_{1}\right)^{1+\frac{2}{\alpha}}}\right\}
\end{aligned}
$$

Proof: The cross entropy between two continuous distributions with PDFs $p(x)$ and $q(x)$ is given by $H(p, q) \triangleq$ $-\int_{0}^{\infty} p(x) \log _{2}(q(x)) \mathrm{d} x$. Therefore, for the case of $\alpha-\eta-\mu$ and $\eta-\mu$ distributions, respectively, it follows that

$$
\begin{aligned}
& H(p, q) \\
& =-\int_{0}^{\infty} \frac{\mathcal{B}_{1} I_{\frac{1}{2}}\left(\mathcal{D}_{1} \gamma^{\frac{\alpha}{2}}\right)}{\gamma^{\frac{1-\alpha \mu}{2}}-\frac{\alpha}{4} e^{\mathcal{C}_{1} \gamma^{\frac{\alpha}{2}}}} \log _{2}\left(\frac{\mathcal{B}_{2} I_{\frac{1}{2}}\left(\mathcal{D}_{2} \gamma\right)}{\gamma^{-\frac{1}{2}} e^{\mathcal{C}_{1} \gamma}}\right) \mathrm{d} \gamma
\end{aligned}
$$

where $\mathcal{B}_{2}=\sqrt{\eta \prime+1} \sqrt{\eta \prime-1} \sqrt{\pi} /\left(\sqrt{\eta} / \sqrt{\bar{\gamma}^{\prime}}\right), \mathcal{C}_{2}=(1+$ $\eta \prime)^{2} /\left(2 \eta / \bar{\gamma}^{\prime}\right)$ and $\mathcal{D}_{2}=(\eta \prime+1)(\eta-1) /\left(2 \eta / \bar{\gamma}^{\prime}\right)$ correspond to the parameters of $\eta-\mu$ distribution. It is evident that the algebraic representation of (8) is similar to (3). Based on this, the proof follows immediately using Lemma 1 .

In the next Section, it is shown that the value of the considered $H(p, q)$ varies substantially at even slight variations of $\alpha$. It is also shown that this variation is larger than that from widely used 
fading parameters such as $\eta$ and $\lambda$. This justifies the usefulness of the $\alpha-\eta-\mu$ and $\alpha-\lambda-\mu$ distributions.

\section{Relative Entropy}

The relative entropy, which is also known as the KullbackLeibler divergence, is a measure of the distance between two probability distributions. It accounts for the inefficiency of assuming that a true distribution with PDF $p(x)$ is replaced by a distribution with PDF $q(x)$. In the present analysis, this measure quantifies the information loss encountered when the non-linearity parameter is not taken into account, which occurs when $\alpha$ $-\eta-\mu$ and $\alpha-\lambda-\mu$ distributions are replaced by the simpler $\eta-\mu$ and $\lambda-\mu$ distributions, respectively.

Lemma 3: For $\left\{\alpha, \eta, \eta \prime, \gamma, \bar{\gamma}, \bar{\gamma}^{\prime}\right\} \in \mathbb{R}^{+}, \mu=1$, the relative entropy between $\alpha-\eta-\mu$ and $\eta-\mu$ models is expressed as

$$
D(p \| q)=H(p, q)-H(p)
$$

where $H(p, q)$ and $H(p)$ are given in (3) and (7), respectively.

Proof: By recalling that the relative entropy is defined as

$$
D(p \| q) \triangleq \int_{0}^{\infty} p(x) \log _{2}\left(\frac{p(x)}{q(x)}\right) \mathrm{d} x
$$

the proof follows with the aid of Lemma 1 and Lemma 2.

Notably, similar expressions for the Shannon entropy of $\alpha$ $-\lambda-\mu$ distribution as well as the cross entropy and relative entropy between $\alpha-\lambda-\mu$ and $\lambda-\mu$ distributions are obtained by setting $\eta=(1-\lambda) /(1+\lambda)$ in (3), (7) and (9), respectively. Extensive results for all considered entropies for both $\alpha-\eta-\mu$ and $\alpha-\lambda-\mu$ fading conditions are provided in Section IV, indicating the non-negligible effects of fading non-linearity.

\section{CAPACITy UNDER DifFERENT AdAPTAtion Policies}

\section{A. Capacity with Optimum Rate Adaptation}

The Shannon capacity of a channel constitutes a theoretical upper bound for the maximum rate of data transmission and is also used as a benchmark for comparisons of the channel capacity under different adaptation schemes [1]. In what follows, we derive a closed-form expression for the average Shannon capacity over $\alpha-\eta-\mu$ fading channels.

Theorem 1: For $\{\alpha, \eta, \bar{\gamma}, B\} \in \mathbb{R}^{+}$and $\mu \in \mathbb{N}$, the average channel capacity over $\alpha-\eta-\mu$ fading channels with optimum rate adaptation is expressed as follows:

$$
\begin{aligned}
& C_{\mathrm{ORA}}^{\alpha-\eta-\mu} \\
& =B \sum_{i=0}^{\mu-1} \frac{\Gamma(\mu+i) \eta^{i} \mu^{\mu-i}(\eta+1)^{\mu-i} \bar{\gamma}^{\frac{\alpha(i-\mu)}{2}}}{2 \ln (2) i ! \Gamma(\mu) \Gamma(\mu-i)(\eta-1)^{\mu+i}}
\end{aligned}
$$

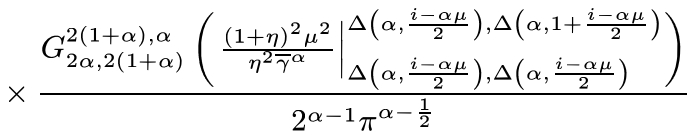

where $B$ is the corresponding bandwidth, $G(\cdot)$ is the Meijer $G$-function and $\Delta(x, y) \triangleq y / x,(y+1) / x, \ldots,(y+x-1) / x$.

Proof: Recalling the definition of the average ergodic capacity

$$
C \triangleq B \int_{0}^{\infty} \log _{2}(1+\gamma) p_{\gamma}(\gamma) \mathrm{d} \gamma
$$

it immediately follows that

$$
C_{\mathrm{ORA}}^{\alpha-\eta-\mu}=B \int_{0}^{\infty} \mathcal{B}_{1} \frac{\ln (1+\gamma) I_{\mu-\frac{1}{2}}\left(\mathcal{D}_{1} \gamma^{\frac{\alpha}{2}}\right)}{\gamma^{\frac{1-\alpha \mu}{2}-\frac{\alpha}{4}} e^{\mathcal{C}_{1} \gamma^{\frac{\alpha}{2}}}} \mathrm{~d} \gamma
$$

With the aid of [33, eq. (8.467)], it follows that

$$
\begin{aligned}
& C_{\mathrm{ORA}}^{\alpha-\eta-\mu} \\
& =\sum_{i=0}^{\mu-1} \int_{0}^{\infty} \frac{(-1)^{i} \mathcal{B}_{1} B \Gamma(\mu+i) \ln (1+\gamma) e^{\mathcal{D}_{1} \gamma^{\frac{\alpha}{2}}} \mathrm{~d} \gamma}{i ! \sqrt{\pi} \Gamma(\mu-i)\left(2 \mathcal{D}_{1}\right)^{i+\frac{1}{2}} \gamma^{1-\frac{\alpha(\mu-i)}{2}} e^{\mathcal{C}_{1} \gamma^{\frac{\alpha}{2}}} .} .
\end{aligned}
$$

Importantly, the exponential and logarithmic terms in (14) can be expressed in terms of the Meijer G-function in [34] yielding

$$
\begin{aligned}
C_{\mathrm{ORA}}^{\alpha-\eta-\mu}= & \sum_{i=0}^{\mu-1} \int_{0}^{\infty} \frac{(-1)^{i} \mathcal{B}_{1} B \Gamma(\mu+i)}{i ! \sqrt{\pi} \Gamma(\mu-i) 2^{i+\frac{1}{2}} \mathcal{D}_{1}^{i+\frac{1}{2}}} \\
& \times \frac{G_{2,2}^{1,2}\left(\left.\gamma\right|_{1,1} ^{1,1}\right) G_{0,1}^{1,0}\left(\left.\mathcal{C}_{1} \gamma^{\frac{\alpha}{2}}\right|_{0} ^{-}\right)}{\gamma^{1-\frac{\alpha(\mu-i)}{2}}} \mathrm{~d} \gamma
\end{aligned}
$$

The above integral can be expressed in closed-form in terms of [34, eq. (21)]. To this effect and after long but basic algebraic manipulations, one obtains (11). This concludes the proof.

Equation (11) is expressed in closed-form and can be computed in software packages such as Maple and Mathematica.

\section{B. Capacity with Optimum Power and Rate Adaptation}

Wireless systems are often subject to average transmit power constraints. Based on this, the maximum capacity for optimum power and rate adaptation, $C_{\mathrm{OPRA}}$, can be determined [1].

Theorem 2: For $\left\{\alpha, \eta, \bar{\gamma}, \gamma_{0}, B\right\} \in \mathbb{R}^{+}$and $\mu \in \mathbb{N}$, the channel capacity over $\alpha-\eta-\mu$ fading channels with optimum power and rate adaptation is expressed as follows:

$$
\begin{aligned}
& C_{\mathrm{OPRA}}^{\alpha-\eta-\mu} \\
& =2 B \sum_{k=0}^{\mu-1} \sum_{i=0}^{\mu-k-1} \frac{(-1)^{k} \eta^{\mu} \Gamma(\mu+k) \Gamma\left(i, \frac{(1+\eta) \mu \gamma_{0}^{\frac{\alpha}{2}}}{\eta \bar{\gamma}^{\frac{\alpha}{2}}}\right)}{k ! i ! \alpha \ln (2) \Gamma(\mu)(\eta-1)^{\mu+k}} \\
& +2 B \sum_{k=0}^{\mu-1} \sum_{i=0}^{\mu-k-1} \frac{(-1)^{\mu} \eta^{k} \Gamma(\mu+k) \Gamma\left(i, \frac{(1+\eta) \mu \gamma_{0}^{\frac{\alpha}{2}}}{\bar{\gamma}^{\frac{\alpha}{2}}}\right)}{k ! i ! \alpha \ln (2) \Gamma(\mu)(\eta-1)^{\mu+k}}
\end{aligned}
$$

where $\gamma_{0}$ is the SNR threshold that determines transmission.

Proof: By firstly recalling the following generic expression

$$
C_{\text {OPRA }} \triangleq B \int_{\gamma_{0}}^{\infty} \log _{2}\left(\frac{\gamma}{\gamma_{0}}\right) p_{\gamma}(\gamma) \mathrm{d} \gamma
$$

as well as substituting (1) and expanding the logarithm using [33, eq. 8.467], one obtains (18) (bottom of the next page). Notably, the two integrals that emerge from the second term of the numerator of (18) can be expressed in terms of [33, eq. (8.310.1)] which yields

$$
\begin{aligned}
& C_{\text {OPRA }}^{\alpha-\eta-\mu} \\
& =\sum_{i=0}^{\mu-1} \frac{B(-1)^{i} \mathcal{B}_{1} \Gamma(\mu+i)}{i ! \sqrt{2 \mathcal{D}_{1} \pi} \Gamma(\mu-i) 2^{i} \mathcal{D}_{1}^{i}} \int_{\gamma_{0}}^{\infty} \frac{\gamma^{\frac{\alpha(\mu-i)}{2}} \ln (\gamma)}{\gamma e^{\gamma^{\frac{\alpha}{2}}\left(\mathcal{C}_{1}-\mathcal{D}_{1}\right)}} \mathrm{d} \gamma \\
& +\sum_{i=0}^{\mu-1} \frac{B(-1)^{\mu} \mathcal{B}_{1} \Gamma(\mu+i)}{i ! \sqrt{2 \mathcal{D}_{1} \pi} \Gamma(\mu-i) 2^{i} \mathcal{D}_{1}^{i}} \int_{\gamma_{0}}^{\infty} \frac{\gamma^{\frac{\alpha(\mu-i)}{2}} \ln (\gamma)}{\gamma e^{\gamma^{\frac{\alpha}{2}}\left(\mathcal{C}_{1}+\mathcal{D}_{1}\right)}} \mathrm{d} \gamma \\
& -B \sum_{i=0}^{\mu-1} \frac{(-1)^{i} \Gamma(\mu+i) \ln \left(\gamma_{0}\right) \Gamma\left(\mu-i,\left(\mathcal{C}_{1}-\mathcal{D}_{1}\right) \bar{\gamma}^{\frac{\alpha}{2}}\right)}{i ! \sqrt{\pi} \alpha \Gamma(\mu-i) \mathcal{B}_{1}^{-1} 2^{i-\frac{1}{2}} \mathcal{D}_{1}^{i+\frac{1}{2}}\left(\mathcal{C}_{1}-\mathcal{D}_{1}\right)^{\mu-i}}
\end{aligned}
$$




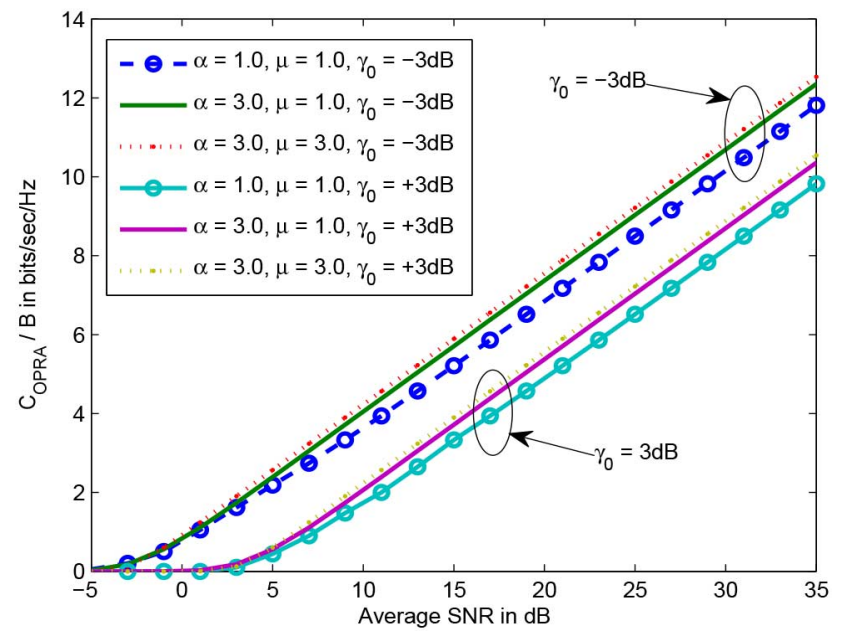

Fig. 1. $C_{\text {OPRA }}$ vs $\bar{\gamma}$ over $\alpha-\eta-\mu$ and $\alpha-\lambda-\mu$ fading channels for $\eta$ $=0.6, \lambda=0.25, \mu=2$ and different values of the fading non-linearity $\alpha$.

Integrating by parts the two remaining integrals and applying [33, eq. (4.352.1)], the resulting integrals can be evaluated using [33, eq. (8.310.1)]. Based on this and after long but basic algebraic manipulations yields (16), completing the proof.

It is noted that closed-form expressions for $C_{\mathrm{ORA}}^{\alpha-\lambda-\mu}$ and $C_{\text {OPRA }}^{\alpha-\lambda-\mu}$ can be readily deduced by setting $\eta=(1-\lambda) /(1+\lambda)$ in (11) and (18). Also, the corresponding channel capacities for $\eta-\mu$ and $\lambda-\mu$ fading channels are deduced for $\alpha=2$.

\section{NumERICAL RESUlts}

The offered results are employed in quantifying the effects of the non-linearity of the propagation medium. To this end, Table I depicts the $H(p), H(p, q)$ and $D(p \| q)$ measures for different values of the fading parameters at $\bar{\gamma}=15 \mathrm{~dB}$. It is shown that the Shannon entropy is inversely proportional to the $\alpha$ parameter which indicates that the more severe the incurred nonlinearity, the more bits are required to encode the corresponding message. To this effect, it is shown that an extra bit is required for encoding the message correctly when $\alpha=1$, with $\eta=2$, $\lambda=1 / 2$ and $\mu=1$, compared to the similar case with $\alpha=3$. On the contrary, it is observed that the lowest value of cross entropy in both cases is for $\alpha=2$, i.e., when $\alpha-\eta-\mu$ and $\alpha-\lambda-\mu$ reduce to $\eta-\mu$ and $\lambda-\mu$, respectively. From this point, the value of $H(p, q)$ increases as the value of $\alpha$ both increases or decreases. For example, while 7 bits/message are practically required when $\alpha=2$, this value increases to $10 \mathrm{bits} / \mathrm{message}$ and $14 \mathrm{bits} / \mathrm{mes}-$ sage for $\alpha=1 / 2$ and $\alpha=3$, respectively. Finally, the relative entropy measure demonstrates the information lost when $\alpha$ is not considered. It is evident that $D(p \| q)=0$ when $\alpha=2$ and increases as $\alpha$ decreases and particularly when it increases.
TABLE I

ENTROPIES OF $\alpha-\eta-\mu$ AND $\alpha-\lambda-\mu$ DistribUtions FOR $\bar{\gamma}=15 \mathrm{~dB}$

\begin{tabular}{|c|c|c|c||c|c|c|c|}
\hline$\alpha-\eta-\mu$ & $\mathrm{H}(\mathrm{p})$ & $\mathrm{H}(\mathrm{p}, \mathrm{q})$ & $\mathrm{D}(\mathrm{p} \| \mathrm{q})$ & $\alpha-\lambda-\mu$ & $\mathrm{H}(\mathrm{p})$ & $\mathrm{H}(\mathrm{p}, \mathrm{q})$ & $\mathrm{D}(\mathrm{p} \| \mathrm{q})$ \\
\hline \hline$\frac{1}{2}-2-1$ & 7.03 & 9.69 & 2.66 & $\frac{1}{2}-\frac{1}{2}-1$ & 6.94 & 9.42 & 2.48 \\
\hline $1-2-1$ & 6.87 & 8.37 & 1.50 & $1-\frac{1}{2}-1$ & 6.86 & 8.21 & 1.36 \\
\hline$\frac{3}{2}-2-1$ & 6.56 & 6.91 & 0.36 & $\frac{3}{2}-\frac{1}{2}-1$ & 6.57 & 6.87 & 0.30 \\
\hline $2-2-1$ & 6.28 & 6.28 & 0.00 & $2-\frac{1}{2}-1$ & 6.31 & 6.31 & 0.00 \\
\hline$\frac{5}{2}-2-1$ & 6.05 & 7.63 & 1.59 & $\frac{5}{2}-\frac{1}{2}-1$ & 6.08 & 7.52 & 1.44 \\
\hline $3-2-1$ & 5.83 & 13.3 & 7.45 & $3-\frac{1}{2}-1$ & 5.88 & 12.5 & 6.62 \\
\hline
\end{tabular}

TABLE II

$C_{\text {ORA }}$ FOR $\alpha-\eta-\mu$ AND $\alpha-\lambda-\mu$ DistriBUTIONS

\begin{tabular}{||c|c||c|c||}
\hline$\alpha-\eta-\mu / \bar{\gamma}(\mathrm{dB})$ & $C_{\mathrm{ORA}}^{\alpha-\eta-\mu} / B$ & $\alpha-\lambda-\mu / \bar{\gamma}(\mathrm{dB})$ & $C_{\text {ORA }}^{\alpha-\lambda-\mu} / B$ \\
\hline \hline $1-1-1 /-5 \mathrm{~dB}$ & 0.458 & $1-0.1-1 /-5 \mathrm{~dB}$ & 0.358 \\
\hline $1-1-1 / 15 \mathrm{~dB}$ & 4.432 & $1-0.1-1 / 15 \mathrm{~dB}$ & 4.428 \\
\hline $1-1-1 / 35 \mathrm{~dB}$ & 10.85 & $1-0.1-1 / 35 \mathrm{~dB}$ & 10.85 \\
\hline $2-1-1 /-5 \mathrm{~dB}$ & 0.529 & $2-0.1-1 /-5 \mathrm{~dB}$ & 0.378 \\
\hline $2-1-1 / 15 \mathrm{~dB}$ & 4.685 & $2-0.1-1 / 15 \mathrm{~dB}$ & 4.674 \\
\hline $2-1-1 / 35 \mathrm{~dB}$ & 11.25 & $2-0.1-1 / 35 \mathrm{~dB}$ & 11.24 \\
\hline $2-2-2 /-5 \mathrm{~dB}$ & 0.385 & $2-0.5-2 /-5 \mathrm{~dB}$ & 0.384 \\
\hline $2-2-2 / 15 \mathrm{~dB}$ & 4.839 & $2-0.5-2 / 15 \mathrm{~dB}$ & 4.819 \\
\hline $2-2-2 / 35 \mathrm{~dB}$ & 11.42 & $2-0.5-2 / 35 \mathrm{~dB}$ & 11.40 \\
\hline $3-2-2 /-5 \mathrm{~dB}$ & 0.398 & $3-0.5-2 /-5 \mathrm{~dB}$ & 0.380 \\
\hline $3-2-2 / 15 \mathrm{~dB}$ & 4.900 & $3-0.5-2 / 15 \mathrm{~dB}$ & 4.886 \\
\hline $3-2-2 / 35 \mathrm{~dB}$ & 11.49 & $3-0.5-2 / 35 \mathrm{~dB}$ & 11.48 \\
\hline $3-3-3 /-5 \mathrm{~dB}$ & 0.399 & $3-0.9-3 /-5 \mathrm{~dB}$ & 0.380 \\
\hline $3-3-3 / 15 \mathrm{~dB}$ & 4.933 & $3-0.9-3 / 15 \mathrm{~dB}$ & 4.888 \\
\hline $3-3-3 / 35 \mathrm{~dB}$ & 11.53 & $3-0.9-3 / 35 \mathrm{~dB}$ & 11.48 \\
\hline \multicolumn{4}{|c|}{}
\end{tabular}

The effect of fading non-linearity on $C_{\text {ORA }}$ and $C_{\text {OPRA }}$ is demonstrated in Table II and Fig. 1, respectively. It is shown that the corresponding spectral efficiency is, as expected, proportional to the value of $\alpha$ in both $\alpha-\eta-\mu$ and $\alpha-\lambda-\mu$ fading conditions. Interestingly, it is shown that the effect of $\alpha$ is more significant than the overall effect of $\eta$ and $\lambda$ parameters together with $\mu$. This illustrates the importance of considering the effects of fading non-linearity in the design and performance analysis of wireless communication systems.

\section{CONCLUSION}

This work quantified the effects of non-linearity, $\alpha$, of the propagation medium in wireless transmission by means of the Shannon entropy, cross entropy and relative entropy measures. The average channel capacity over generalized non-linear fading conditions was also evaluated for the cases of optimum rate and optimum power and rate adaptations. It was shown that the effects of $\alpha$ are more pronounced than those of the commonly used fading parameters $\eta, \lambda$ and $\mu$ combined.

$$
\begin{aligned}
C_{\mathrm{OPRA}}^{\alpha-\eta-\mu} & =\sum_{i=0}^{\mu-1} \int_{\gamma_{0}}^{\infty} \frac{\ln (\gamma)\left[(-1)^{i} e^{-\gamma^{\frac{\alpha}{2}}\left(\mathcal{C}_{1}-\mathcal{D}_{1}\right)}+(-1)^{\mu} e^{-\gamma^{\frac{\alpha}{2}}\left(\mathcal{C}_{1}+\mathcal{D}_{1}\right)}\right]-\ln \left(\gamma_{0}\right)\left[(-1)^{i} e^{-\gamma^{\frac{\alpha}{2}}\left(\mathcal{C}_{1}-\mathcal{D}_{1}\right)}+(-1)^{\mu} e^{-\gamma^{\frac{\alpha}{2}}\left(\mathcal{C}_{1}+\mathcal{D}_{1}\right)}\right]}{i ! \sqrt{\pi} \Gamma(\mu-i) 2^{i+\frac{1}{2}} \mathcal{D}_{1}^{i+\frac{1}{2}} B^{-1} \mathcal{B}_{1}^{-1} \gamma^{1-\frac{\alpha(\mu-i)}{2}}[\Gamma(\mu+i)]^{-1}} \mathrm{~d} \gamma \\
& -B \sum_{i=0}^{\mu-1} \frac{(-1)^{i} \Gamma(\mu+i) \ln \left(\gamma_{0}\right) \Gamma\left(\mu-i,\left(\mathcal{C}_{1}+\mathcal{D}_{1}\right) \bar{\gamma}^{\frac{\alpha}{2}}\right)}{i ! \sqrt{\pi} \alpha \Gamma(\mu-i) \mathcal{B}_{1}^{-1} 2^{i-\frac{1}{2}} \mathcal{D}_{1}^{i+\frac{1}{2}}\left(\mathcal{C}_{1}+\mathcal{D}_{1}\right)^{\mu-i}}
\end{aligned}
$$




\section{REFERENCES}

[1] M. K. Simon and M.-S. Alouini, Digital Communication Over Fading Channels. New York, NY, USA: Wiley, 2005.

[2] M. D. Yacoub, "The $\alpha-\mu$ distribution: A physical fading model for the Stacy distribution," IEEE Trans. Veh. Technol., vol. 56, no. 1, pp. 27-34, Jan. 2007.

[3] P. C. Sofotasios, "On special functions and composite statistical distributions and their applications in digital communications over fading channels," Ph.D. dissertation, Univ. of Leeds, Leeds, U.K., 2010.

[4] D. Benevides da Costa and M. D. Yacoub, "Average channel capacity for generalized fading scenarios," IEEE Commun. Lett., vol. 11, no. 12, pp. 949-951, Dec. 2007.

[5] D. Benevides da Costa, M. D. Yacoub, and J. C. S. S. Filho, "Highly accurate closed-form approximations to the sum of $\alpha-\mu$ variates and applications," IEEE Trans. Wireless Commun., vol. 7, no. 9, pp. 3301-3306, Sep. 2008.

[6] P. C. Sofotasios and S. Freear, "The $\alpha-\kappa-\mu /$ gamma composite distribution: A generalized non-linear multipath/shadowing fading model," in Proc. IEEE INDICON '11, Hyderabad, India, Dec. 2011, pp. 1-6.

[7] P. C. Sofotasios and S. Freear, "The $\alpha-\kappa-\mu$ extreme distribution: Characterizing non linear severe fading conditions," in Proc. ATNAC '11, Melbourne, Australia, Nov. 2011

[8] D. Zogas and G. K. Karagiannidis, "Infinite-series representations associated with the bivariate Rician distribution and their applications," IEEE Trans. Commun., vol. 53, no. 11, pp. 1790-1794, Nov. 2005.

[9] P. C. Sofotasios and S. Freear, "The $\eta-\mu /$ gamma composite fading model," in Proc. IEEE ICWITS '10, Honolulu, HI, USA, Aug. 2010, pp. 1-4.

[10] P. C. Sofotasios and S. Freear, "The $\eta-\mu /$ gamma and the $\lambda-\mu /$ gamma multipath/shadowing distributions," in Proc. ATNAC '11, Melbourne, Australia, Nov. 2011.

[11] P. C. Sofotasios and S. Freear, "The $\kappa-\mu /$ gamma composite fading model," in Proc. IEEE ICWITS '10, Honolulu, HI, USA, Aug. 2010, pp. 1-4.

[12] E. J. Leonardo, D. Benevides da Costa, U. S. Dias, and M. D. Yacoub, "The ratio of independent arbitrary $\alpha-\mu$ random variables and its application in the capacity analysis of spectrum sharing systems," IEEE Commun. Lett., vol. 16, no. 11, pp. 1776-1779, Nov. 2012.

[13] P. C. Sofotasios and S. Freear, "The $\kappa-\mu /$ gamma extreme composite distribution: A physical composite fading model," in Proc. IEEE WCNC '11, Cancun, Mexico, Mar. 2011, pp. 1398-1401.

[14] P. C. Sofotasios and S. Freear, "On the $\kappa-\mu$ /gamma composite distribution: A generalized multipath/shadowing fading model," in Proc. IEEE IMOC '11, Natal, Brazil, Oct. 2011, pp. 390-394.

[15] D. S. Michalopoulos, G. K. Karagiannidis, T. A. Tsiftsis, and R. K. Mallik, "Wlc41-1: An optimized user selection method for cooperative diversity systems," in Proc. IEEE GLOBECOM '06, 2006, pp. 1-6.

[16] P. C. Sofotasios, M. Valkama, T. A. Tsiftsis, Y. A. Brychkov, S. Freear, and G. K. Karagiannidis, "Analytic solutions to a marcum $Q$-integral and application in energy detection of unknown signals over multipath fading channels," in Proc. 9th CROWNCOM '14, Oulu, Finland, Jun. 2-4, 2014, pp. 260-265.

[17] N. D. Chatzidiamantis and G. K. Karagiannidis, "On the distribution of the sum of gamma-gamma variates and applications in RF and optical wireless communications," IEEE Trans. Commun., vol. 59, no. 5, pp. 1298-1308, May 2011.
[18] P. C. Sofotasios, T. A. Tsiftsis, M. Ghogho, L. R. Wilhelmsson, and M. Valkama, "The $\eta-\mu$ /inverse-Gaussian Distribution: A novel physical multipath/shadowing fading model," in IEEE ICC '13, Budapest, Hungary, Jun. 2013, pp. 5715-5719.

[19] S. Harput, P. C. Sofotasios, and S. Freear, "A novel composite statistical model for ultrasound applications," in Proc. IEEE IUS '11, Oct. 8-10, 2011, pp. 1-4.

[20] E. J. Leonardo and M. D. Yacoub, "The product of two $\alpha-\mu$ variates and the composite $\alpha-\mu$ multipath-shadowing model," IEEE Trans. Veh. Technol, vol. 64, no. 6, pp. 2720-2725, Jun. 2015.

[21] P. C. Sofotasios, T. A. Tsiftsis, K. Ho-Van, S. Freear, L. R. Wilhelmsson, and M. Valkama, "The $\kappa-\mu$ /inverse-Gaussian composite statistical distribution in RF and FSO wireless channels," in Proc. IEEE VTC '13 - Fall, Las Vegas, NV, USA, Sep. 2013, pp. 1-5.

[22] N. Y. Ermolova and O. Tirkkonen, "The $\eta-\mu$ fading distribution with integer values of $\mu$," IEEE Trans. Wirel. Commun., vol. 10, no. 6, pp. 1976-1982, Jun. 2011.

[23] P. S. Bithas, N. C. Sagias, P. T. Mathiopoulos, and G. K. Karagiannidis, "On the performance analysis of digital communications over generalized $-K$ fading channels," IEEE Commun. Lett., vol. 10, no. 5, pp. 353-355, May 2006.

[24] P. S. Bithas, G. P. Efthymoglou, and N. C. Sagias, "Spectral efficiency of adaptive transmission and selection diversity on generalized fading channels," IET Commun., vol. 4, no. 17, pp. 2058-2064, 2010.

[25] G. Fraidenraich and M. D. Yacoub, "The $\alpha-\eta-\mu$ and $\alpha-\kappa-\mu$ fading distributions," in Proc. IEEE ISSSTA '06, Manaus, Brazil, 2006.

[26] A. K. Papazafeiropoulos and S. A. Kotsopoulos, "The $\alpha-\eta-\mu$ and $\alpha-\lambda-\mu$ joint envelope-phase fading distributions," in Proc. IEEE ICC '09, Dresden, Germany, Jun. 2009, pp. 919-922.

[27] A. Papazafeiropoulos and S. A. Kotsopoulos, "The $\alpha-\lambda-\mu$ and $\alpha-\eta$ $-\mu$ small-scale general fading distributions: A unified approach," in Wireless Personal Communications. : Springer, Nov. 2009.

[28] A. Papazafeiropoulos and S. A. Kotsopoulos, "Statistical properties for the envelope and phase of the $\alpha-\eta-\mu$ generalized fading channels," in Wireless Personal Communications. New York, NY, USA: Springer, Jul. 2011.

[29] P. C. Sofotasios, T. A. Tsiftsis, Y. A. Brychkov, S. Freear, M. Valkama and G. K. Karagiannidis, "Analytic expressions and bounds for special functions and applications in communication theory," IEEE Trans. Inf Theory, vol. 60, no. 12, pp. 7798-7823, Dec. 2014.

[30] R. Cogliatti and R. A. A. de Souza, "A near - 100\% efficient algorithm for generating $\alpha-\kappa-\mu$ and $\alpha-\eta-\mu$ variates," in Proc. IEEE VTC '13, Las Vegas, NV, USA, Sep. 2013.

[31] E. Salahat and A. Hakam, "Performance analysis of $\alpha-\eta-\mu$ and $\alpha-\kappa-\mu$ generalized mobile fading channels," in Proc. Eur. Wireless '14, Barcelona, Spain, May 16-18, 2014, pp. 992-997.

[32] E. Salahat and A. Hakam, "Novel unified expressions for error rates and ergodic channel capacity analysis over generalized fading subject to AWGGN," in Proc. Proc. IEEE Globecom '14, Austin, TX, USA, Dec. 8-12, 2014, pp. 3976-3982.

[33] I. S. Gradshteyn and I. M. Ryzhik, Tables of Integrals Series, and Products, 7th ed. New York, NY, USA: Academic, 2007.

[34] V. S. Adamchik and O. I. Marichev, "The algorithm for calculating integrals of hypergeometric functions and its realization in reduce system," in Proc. ICSAC '90, Tokyo, Japan, 1990, pp. 212-224. 